\title{
Excretion of Dicarboxylic and $\omega-1$ Hydroxy Fatty Acids by Low Birth Weight Infants Fed with Medium-Chain Triglycerides
}

\author{
R. K. WHYTE, D. WHELAN, R. HILL, AND S. MCCLORRY \\ Departments of Pediatrics and Pathology, McMaster University Medical Centre, Hamilton, Ontario, Canada
}

\begin{abstract}
The effects of feeding a commercial formula containing increased amounts of medium-chain fatty acids on the urinary excretions of $\mathrm{C} 6$-C10 moncarboxylic, dicarboxylic, and $\omega-1$ hydroxy acids were studied in 13 growing preterm infants of mean birth weight $1.42 \mathrm{~kg}$ in a randomized double-blind cross-over clinical trial. Infants were allocated to two sequential feeding regimes of 5 days each, during the last 3 days of which urine was collected and analysed by gas-chromatography mass spectrometry. The two feeding regimes consisted of high energy and proteincontaining formulas specially designed for growing low birth weight infants. In one diet the triglyceride component consisted of $46 \%$ medium-chain (C8-C10) and 54\% longchain fatty acid residues: in the other it consisted of $4 \%$ medium-chain and $96 \%$ fatty acid residues. The infants were randomized so that six infants were fed first with the medium-chain predominant formula and seven were fed first with the long-chain predominant formula. There were significant differences in the urinary excretions of octanoate, sebacate, suberate, adipate, 7-hydroxyoctanoate, and 5-hydroxyhexanoate; these substances appearing in much greater quantities in the urine during the period in which medium-chain triglycerides were predominant in the formula. The significance of this organic aciduria, which accounted for $0.7 \%$ of the dietary intake of medium-chain triglycerides, remains to be established. (Pediatr Res 20: 122-125, 1986)
\end{abstract}

Medium-chain triglycerides, which are fats containing fatty acid residues of chain length $\mathrm{C} 8-\mathrm{C} 10$, are now incorporated into formulas designed to increase the energy intake of growing low birth weight infants. Energy is a rate-limiting factor for growth of the low birth weight infant (1), and fat is the most energy dense form of food. The newborn infant (2), and particularly the low birth weight infant (3), does not absorb fat well by adult standards. A number of modifications of cow's milk-based infant formulas have been introduced to improve the digestibility of fat, including the substitution of vegetable oils for animal fats ( 3 , 4). Tantibhedyankul and Hashim (5) claimed that the partial substitution of medium-chain for long-chain triglycerides in the diets of low birth weight infants could increase the coefficient of digestibility for fat from 76 to $97 \%$. This observation led to the development of special formulas for the low birth weight infant in which long-chain triglycerides were partially or completely

Received March 28.1985; accepted September 16.1985.

Correspondence to R. K. Whyte. B.Sc. MB. MRCP (UK). FRCP (C), Department of Pediatries. McMaster University, 1200 Main St. West, Hamilton, Ontario L8N 3 Z5 Canada.

This study was funded by Ross Laboratories. Columbus. $\mathrm{OH}$ who also prepared and analyzed the experimental formulas. replaced by medium-chain triglycerides. While the digestibilities of these fats are greater than their long-chain triglyceride equivalents, the rates, sites, and modes of metabolism of these two forms of fatty acids are quite different (6). Long-chain fatty acids, after intestinal hydrolysis and reesterification, are distributed to the body by the lymphatic system and subsequently hydrolyzed by tissue lipoprotein lipase, with only a proportion being presented to the liver. Medium-chain fatty acids are hydrolyzed in the intestine to medium-chain fatty acids, and are subsequently taken up rapidly in the mesenteric venous system to enter the hepatic system directly in the portal venous blood; very little medium-chain fatty acid reaches the extrahepatic tissues (7).

In the hepatocyte long-chain acids are only slowly acylated at a rate limited by the kinetics of binding to fatty acid binding protein, while medium-chain triglycerides are immediately acylated on entry to the hepatocyte (8). Oxidation of long chain fatty acids depends on entry to the mitochondrion, which in turn depends upon the integrity of the carnitine system; mediumchain fatty acids can enter the mitochondrion directly without the involvement of these intermediates (9). The differences between long and medium chain triglycerides, therefore, go beyond simple differences in digestibility.

We have conducted a randomized controlled clinical trial in which growing low birth weight infants were fed high energycontaining formulas of which the fat content consisted of either $46 \%$ medium- and $54 \%$ long-chain triglycerides or of $4 \%$ medium- and $96 \%$ long-chain triglycerides. Early in the trial some unusual organic acids were detected in the urines of infants recruited to the trial; we therefore undertook to ask if differences in urinary excretions of organic acids in low birth weight infants were caused by differing dietary intakes of medium-versus longchain fatty acids.

\section{SUBJECTS, MATERIALS, AND METHODS}

Thirteen growing low birth weight infants were studied. Infants were eligible for the study if they were of birth weight less than $1800 \mathrm{~g}$, were free of respiratory, infectious, or gastrointestinal disease, and were growing, as evidenced by 3 consecutive days of weight gain. All infants were enterally fed and receiving approximately $150 \mathrm{ml} / \mathrm{kg}$. day of Special Care formula (Ross Laboratories, Columbus, $\mathrm{OH}$ ) on entry to the study. Informed consent was obtained from the infant's parents.

Two experimental formulas were prepared by a formula manufacturer (Ross Laboratories) so that they were identical in protein and energy content but different in fat composition. One formula (A) was commercial Special Care Formula (10) in which medium-chain triglycerides comprised $46 \%$ (by weight) of the total fat content; the remainder were long-chain triglycerides of vegetable origin. The other (B) had a fat composition which was characteristic of a regular infant formula in that $4 \%$ of the fat was present as medium-chain triglycerides. The macronutrient 
Table 1. Macronutrient and fatty acid composition of formulas

\begin{tabular}{|c|c|c|c|}
\hline & & Diet A & Diet B \\
\hline & & \multicolumn{2}{|c|}{ per liter } \\
\hline Protein & $\mathrm{g}$ & 21.4 & 21.9 \\
\hline Total Fat & $\mathrm{g}$ & 44.1 & 43.5 \\
\hline $\begin{array}{l}\text { as medium-chain tri- } \\
\text { glycerides }\end{array}$ & $\mathrm{g}$ & 20.5 & 1.8 \\
\hline $\begin{array}{l}\text { as long-chain triglyc- } \\
\text { erides }\end{array}$ & $\mathrm{g}$ & 23.6 & 41.7 \\
\hline Carbohydrate & $\mathrm{g}$ & 83.3 & 81.6 \\
\hline \multicolumn{4}{|c|}{$\%$ of total fatty acids by nt } \\
\hline C6:0 & Caproate & 0.2 & 0.0 \\
\hline C $8: 0$ & Caprylate & 29.4 & 2.2 \\
\hline $\mathrm{C} 10: 0$ & Caprate & 16.2 & 1.8 \\
\hline C 12:0 & Laurate & 8.1 & 14.7 \\
\hline C $14: 0$ & Myristate & 3.5 & 6.6 \\
\hline $\mathrm{C} 16: 0$ & Palmitate & 6.3 & 10.4 \\
\hline C18:0 & Stcarate & 2.4 & 3.1 \\
\hline $\mathrm{C} 18.1$ & Oleate & 10.7 & 18.9 \\
\hline C18:2 & Linoleate & 22.0 & 41.1 \\
\hline $\mathrm{C} 18: 3$ & Linolenate & 1.0 & 0.7 \\
\hline C20:4 & Arachidonate & 0.2 & 0.2 \\
\hline
\end{tabular}

content and composition of the fats in the two formulas according to the manufacturer's analysis is given in Table 1. The formulas were identical in mineral, trace element, and vitamin content, which was identical to that of commercial Special Care formula (10). The formula labels were coded by the manufacturer so that their identities were not known to the investigators.

Each infant was fed with each formula for a 5-day period, making a total study period of 10 days. The order in which the infants were fed each formula was randomized using random number tables and sealed cards. Infants were fed by gavage from preweighed and shaken bottles, so that the planned total daily intake of formula was $150 \mathrm{~g} / \mathrm{kg}$. day; the actual feed intake was measured by weighing the bottle after the feed. For the final 3 days of each 5-day period 24-h collections of urine were made through improvized adhesive rubber finger stalls connected to continuous drainage. The urines were pooled as 3 -day collections and frozen at $-20^{\circ} \mathrm{C}$.

For analysis the urine was thawed to room temperature and an internal standard [tropic acid (2-phenyl-3-hydroxypropionic)] was added to a 2-ml sample, to a final concentration of $50 \mu \mathrm{mol} /$ liter. Extraction and derivitization was as previously described (11). To limit adsorptive losses all glassware used in the extraction procedure was siliconized by prior treatment with Aquasil (Pierce, Rockford, IL). A urine standard containing known amounts of organic acids was analyzed together with each batch of unknown samples.

One and one-half $\mu \mathrm{l}$ of the derivitized extract, together with $1.5 \mu \mathrm{l}$ of n-octane, were injected into the gas chromatography/ mass spectrometry system (Finnigan $4000 \mathrm{GC} / \mathrm{MS}$, with Incos Data System, Finnigan-Matt, Sunnyvale, CA). The analytical column was a fused silica open tubular capillary $(30 \mathrm{~m} \times 0.21$ $\mathrm{mm}$ ID), with a $1 \mu \mathrm{m}$ bonded methylsilicone stationary phase (DBI, J and W Scientific, Rancho Cordoba, CA). The injection was "on column." The carrier gas was helium (linear velocity 30 $\mathrm{cm} / \mathrm{s}$ ). The temperature programme was $90^{\circ} \mathrm{C}$ for $4 \mathrm{~min}$, then $2-5^{\circ} \mathrm{C} / \mathrm{min}$ to $250^{\circ} \mathrm{C}$. The column was interfaced directly into the ion source. Ionization was by electron impact ( $70 \mathrm{ev}$, emission current $0.35 \mathrm{~mA}$ ). The ion source was at $280^{\circ} \mathrm{C}$, the electron multiplier at $-1250 \mathrm{~V}$. Mass spectra were recorded continuously between 60 and 500 amu (1-2 s/upward scan), and the data collected and processed by the Incos system. The concentrations of the respective organic acids were determined via the Finnigan Target Compound Analysis software of this data system. Briefly this software allows for the integration of the peak area due to the ion current of a selected mass for each of the organic acids and for the internal standard. The calculation of the ratios: peak area organic acid/peak area internal standard in the unknowns and standard allow for the quantitation of the respective organic acids by the simple formula:

concentration in the unknown

$$
=\frac{\text { concentration in standard } \times \text { ratio in unknown }}{\text { ratio in standard }}
$$

Statistical analysis of differences in the urinary organic acid concentrations between the two randomized groups was by unpaired $t$ test; differences within subjects and between dietary regimes were compared by the Mann-Whitney U test. Stepwise multiple regression analysis was performed to examine correlations between organic acid excretions, using an F-to-enter value of 4.84 (for $n=13, p=0.05$ ).

\section{RESULTS}

All of the 13 infants enrolled completed the study. Six infants received formula $\mathrm{A}$ first, and seven formula $\mathrm{B}$, so that in all there was a total of 26 balance periods, with 13 allocated to each diet. There were no significant differences between those infants who received formula $\mathrm{A}$ or formula $\mathrm{B}$ first in birthweight (mean 1.42 $\mathrm{kg}$, SD 0.33), gestational age (mean 31 weeks, SD 2), or postnatal age (13 days, SD 7) on entry to the study. When comparisons were made between the balance periods on each diet there were again no significant differences in body weights, daily feed intakes, or urine volumes (Table 2).

Urine excretions of organic acids were very different between dietary groups. Daily urinary excretions of organic acids during each of the diets are given in Table 2. There were no significant differences in the daily urine outputs or in excretion of urinary lactate or 4-hydroxyphenylacetate. There was a 2-fold increase in succinate excretion with diet A compared to diet $\mathrm{B}(p<0.05)$. Large and significant differences were observed in excretions of octanoate, the C10-, C8-, and C6-dicarboxylic acids (respectively, sebacate, suberate, and adipate), and in the $\omega-1$ hydroxylated medium- and short-chain acids 7-hydroxyoctanoate and 5-hydroxyhexanoate. Two of these fatty acid derivatives (sebacate and 5-hydroxyhexanoate) were not identified at all in the urines during the diet $\mathrm{B}$ balance periods.

Table 2. Body wt, feed intake, and urinary excretion of organic acids by infants fed diets high $(A)$ or low $(B)$ in medium-chain triglyceride content

\begin{tabular}{lcccccc}
\hline & \multicolumn{2}{c}{ Diet A } & & \multicolumn{2}{c}{ Diet B } & \\
\cline { 2 - 3 } \cline { 5 - 6 } & Median & Range & & Median & Range & \\
\hline Body w1 & 1.62 & $0.92-2.36$ & & 1.71 & $1.08-2.08$ & NS \\
Feed intake (g/kg.day) & 156 & $147-161$ & & 157 & $147-166$ & NS \\
Urine output (ml/ & 83 & $55-90$ & & 76 & $51-92$ & NS
\end{tabular}

kg.day)

\begin{tabular}{lrcrrr}
\multicolumn{7}{c}{$\mu m o l / k g . d a y$} \\
Lactate & 23.4 & $1.4-94.4$ & 35.6 & $0.4-75.5$ & NS \\
4-Hydroxyphenylacetate & 11.3 & $2.5-49.9$ & 9.1 & $0.7-21.2$ & NS \\
Succinate & 56.2 & $37.0-380.0$ & 46.5 & $0.0-221.1$ & $*$ \\
Octanoate & 3.2 & $0.0-10.3$ & 0.1 & $0.0-0.5$ & $\dagger$ \\
Adipate & 15.9 & $4.7-56.1$ & 1.1 & $0.0-2.9$ & $\dagger$ \\
Suberate & 29.0 & $8.0-82.9$ & 0.6 & $0.0-1.1$ & $\dagger$ \\
Scbacate & 66.7 & $8.7-172.6$ & 0.0 & $0.0-0.0$ & $\dagger$ \\
5-Hydroxyhexanoate & 5.4 & $0.0-28.5$ & 0.0 & $0.0-0.0$ & $\dagger$ \\
7-Hydroxyoctanoate & 7.3 & $0.0-17.2$ & 0.0 & $0.0-1.6$ & $\dagger$
\end{tabular}

$* p<0.05$.

$+p<0.0005$. 
Table 3. Stepwise multiple regression analvsis of organic acid excretion

\begin{tabular}{lll}
\hline Predicted & Predicting variables & $r$ \\
\hline 5-OH hexanoate & Octanoate & 0.77 \\
7-OH octanoate & Octanoate & 0.72 \\
Octanoate & Adipate & 0.81 \\
Adipate & Subcrate & 0.88 \\
Scbacate & Lactate & 0.57 \\
\hline
\end{tabular}

During diet A a number of correlations between excretion of the organic acids were discernible (Table 3). Stepwise multiple regression analysis resulted in the selection of only one significant predictive variable for each organic acid daily excretion. The daily excretion of octanoate was the most powerful predictor of both 7-hydroxyoctanoate and of 5-hydroxyhexanoate excretion. Similar relationships were demonstrable between excretion of adipate and octanoate, and between suberate and adipate. The daily excretion of sebacate was not associated with that of any of the other dicarboxylic acids, with the $\omega-1$ hydroxyacids or with octanoate excretion. During diet B there were no such relationships between organic acid excretion.

There were no detectable quantities of decanoate, 9-hydroxydecanoate. hexanoate, acetoacetate, or of $\beta$-hydroxybutyrate in urines from either dietary regime.

\section{DISCUSSION}

Feeding low birth weight infants with a commercial formula high in medium-chain triglycerides causes an organic aciduria featuring unusual dicarboxylic and $\omega$-1-hydroxy fatty acids. This phenomenon has been previously described by Shigematsu et al. (12) in seven low birth weight infants fed with formula in which $75 \%$ of the fat was present as medium-chain triglycerides. In that study, which was not controlled with a standard formula group, quantities of $\omega$-1-hydroxy and dicarboxylic acids of chain length C6-C10 were identified, along with substantial quantities of sebacate; 9-hydroxydecanoate was not detected.

Our study was carried out as a randomized cross-over clinical trial; we have demonstrated that the urinary organic acids identified are caused by the difference in diets and not by some physiologic peculiarity of prematurity. Our findings are similar to those of Shigematsu et al. (12) in that the relative concentrations of dicarboxylic and $\omega-1$ hydroxy fatty acids were similar, and that despite large dietary loads of decanoate no detectable quantities of 9-hydroxydecanoate were found. Further comparison is difficult because Shigematsu et al. (12) do not give details of the weights of their infants and the fatty acid composition of their formulas were quite different from ours.

The excretion of dicarboxylic acids in response to high levels of dietary medium-chain triglycerides is not a phenomenon confined to the newborn or to low birth weight infants. Urinary excretion of dicarboxylic acids was first described in 1934 in two adults receiving trioctanoate (13); since then Mortensen and Gregersen (14) described the finding in four children aged between 2 and 9 months being fed with a formula containing medium-chain triglyceride and in a 28 -year-old man ingesting medium-chain triglyceride oil. Their findings differ from ours in that $\omega-1$ hydroxyacid excretion was not described, but, in common with our findings, they demonstrated a progressive increase in excretion of dicarboxylic acids with increasing chain length from adipate to sebacate. This progression is reversed in patients with nonketotic C6-C10-dicarboxylic aciduria [who also excrete 5-hydroxyhexanoate (15)] and it can be used to identify such cases from infants receiving medium-chain triglycerides (14).

$\Lambda$ part from introducing diagnostic confusion with inborn errors of metabolism, such as non-ketotic C6-C10-dicarboxylic aciduria (15), glutaric aciduria type II (16), ethylmalonic adipate aciduria (17), and riboflavin responsive multiple acyl-CoA de- hydrogenase defect (18), the pathologic significance of the excretion of dicarboxylic and $\omega-1$ hydroxyacids remains to be determined. Our experience suggests that the phenomenon is likely to be common in low birth weight infants receiving specialized "low birth weight formulas" in which large quantities of fat are present as medium-chain triglycerides. According to Gregerson et al. (19), the $\omega$ - and $\omega-1$ oxidation pathways are catalyzed by enzyme systems comprised of cytochrome P-450-dependent monooxygenase localized in the microsomes. In their studies of the affinities of these oxidation pathways for the monocarboxylic acids $\mathrm{C} 6-\mathrm{C} 12$, they conclude that $\mathrm{C} 8$ - and $\mathrm{C} 6$-dicarboxylic acids excreted by patients with acyl-CoA dehydrogenation deficiencies are derived by $\beta$-oxidation of sebacic and dodecanedioic acyl $\mathrm{CoA}$ residues rather than by direct oxidation of octanoic and hexanoic residues, and that the $\omega-1$ hydroxylated $\mathrm{C} 8-\mathrm{C} 10$ acids are derived by $\beta$-oxidation of 11-hydroxydodecanoic residues. The $\mathrm{Km}$ for the $\omega-1$ oxidation of decanoate is particularly high (19); this is consistent with the finding that 9-hydroxydecanoate did not appear in the urine of such patients and was not detectable in our study. Infants in our study received high intakes of medium-chain triglycerides from diet $\mathrm{A}$, which would yield 13 $\mathrm{mmol} / \mathrm{kg}$. day of octanoate and $6 \mathrm{mmol} / \mathrm{kg} \cdot$ day $(2.1 \mathrm{~g})$ of decanoate; in these circumstances it is likely that some direct oxidation of both octanoate and decanoate took place. Regression analysis indicated that while there were high correlations between excretions of octanoate, adipate, suberate, and $\omega-1$ hydroxy octanoate and hexanoate, there was little correlation between these daily excretions and that of sebacate, suggesting indirectly that the biosynthesis of this acid was independent of the shorter chain forms. Dodecanoate (laurate), which for the purposes of this study was classed as a long-chain triglyceride, was present in greater amounts in diet B (Table 1). It is therefore unlikely that $\beta$-oxidation of 11-hydroxydodecanoate gave rise to the higher observed excretions of $\mathrm{C} 6-\mathrm{C} 10$ dicarboxylic and $\omega-1$ hydroxy acids with diet $B$. As hexanoate was present in diet A in very small amounts, the adipate and 5-hydroxyhexanoate may have come from either the $\omega-1$ oxidation of hexanoyl-CoA or from the $\beta$-oxidation of 7-hydroxyoctanoate.

Clearly the urinary excretion of oxidized forms of mediumand short-chain fatty acids is causally related to a diet that is high in medium-chain triglycerides. Gregersen et al. (19) consider the cytochrome-P450-dependent oxidation to be a detoxification mechanism for hexanoate, octanoate, and decanoate. In infants in whom acyl dehydrogenation is limited by inborn errors of metabolism (17), large doses of medium-chain triglycerides precipitate coma, with excretion of $\omega-1$ hydroxy and dicarboxy fatty acids. Unlike the normal preterm infants in this study, such infants also demonstrate substantial ketoacidosis. Urinary excretion of dicarboxylic acids is seen in Jamaican vomiting sickness, and $\omega-1$ hydroxyaciduria has been associated with an idiopathic form of hypoglycaemia (21), but the presence of these acids themselves has not been shown to be causative of hypoglycemia or any other metabolic disturbance. Of the $19 \mathrm{mmol} / \mathrm{kg} \cdot$ day of medium-chain triglyceride given in diet $\mathrm{A}$, about $130 \mu \mathrm{mol} / \mathrm{kg}$. day or $0.7 \%$ were excreted as urinary organic acids. In terms of energy balance, the excretory loss of medium-chain triglyceride is small. There is therefore no evidence that the presence of these oxidation products of medium-chain triglyceride metabolism represent an adverse effect of a diet high in medium-chain triglycerides; their origins and significance remain to be determined.

\section{REFERENCES}

1. Whyte RK, Haslam R, Vlainic C, Shannon S, Samulski K. Campbell D, Bayley HS. Sinclair JC 1983 Energy balance and nitrogen balance in growing infants fed human milk or formula. Pediatr Res 17:891-898

2. Bach A. Phan T, Metais P 1976 Effect of the fatty acid composition of ingested fats on rat liver intermediatry metabolism. Horm Metab Res 8:375-379

3. Tidwell HC. Holt LE. Farrow HL. Neale S 1935 Studies in fat metabolism II. Fat absorption in premature infants and twins. J Pediatr 6:481-489 
4. Holt LE, Tidwcll HC, Kirk CM. Cross DM, Neale S. 1935 Studies in fat metabolism. I. Fat absorption in normal infants. J Pcdiatr 6:427-480

5. Tantibhedyankul P. Hashim SA 1971 Clinical and physiologic aspects of mcdium chain triglycerides: alleviation of steatorrhea in premature infants. Bull NY Acad Med 47:17-33

6. Bach AC, Babayan VK 1982 Medium chain triglycerides: an update. Am J Clin Nutr 36:950-96?

7. Hashim SA 1968 Studies of medium chain fatty acid transport in portal blood. In: Senior JR (ed) Medium Chain Triglycerides. University of Pennsylvania Press, Philadelphia. pp 81-90

8. Wu-Rideout MYC. Elson C. Schrago E 1976 The role of fatty acid binding protein on the metabolism of fatty acids in isolated rat hepatocytes. Biochem Biophys Res Commun 809-816

9. Bremer $\mathbf{J} 1980$ Camitine and its role in fatty acid metabolism. Trends Biochem Sci 2:207-209

10. Similac ${ }^{(R)}$ Infant Formulas 1984. Ross Laboratories, Montreal, Canada

11. Whelan DT. Hill RE. McClorry S 1983 Fumaric aciduria: a new organic aciduria, associated with mental retardation and speech impairment. Clin Chim Acta 132:301-308

12. Shigematsu Y. Momoi T. Sudo M. Suzuki Y 1981 ( $\omega$-1)-Hydroxymonocarboxylic acids in urine of infants fed medium-chain triglycerides. Clin Chem 27:1661-1664

13. Verkade PE van der Lee J 1934 VI. Researches on fat metabolism II. Biochem J $28: 31-40$

14. Mortensen PE, Gregersen N 1980 Medium chain triglyceride medication as a pitfall in the diagnosis of non-ketotic C6-C10-dicarboxylic acidurias. Clin Chim Acta 103:33-37

15. Gregersen N, Rosleff F. Kolvraa S, Holboth N, Rasmussen K, Lauritzen R 1980 Non-Ketotic C6-C10-dicarboxylic aciduria: biochemical investigations of two cases. Clin Chem Acta 102:179-189

16. Przyrembel H, Wendel U, Becker K. Bremer HJ, Bruinvis L, Ketting D, Wadman SK 1976 Glutaric aciduria type II: report on a previously undescribed metabolic disorder. Clin Chem Acta 66:227-239

17. Mantagos S, Genel M. Tanaka K 1979 Ethylmalonic adipic aciduria: in vivo and in vitro studics indicating deficiency of activities of multiple acyl-CoA dehydrogenase. J Clin Invest 64:1580-1589

18. Gregersen N, Wurzensen H, Kolvraa S, Christensen E. Christensen MA, Brandt NJ, Rasmussen K 1982 C6-C10-dicarboxylic aciduria: investigation of a patient with riboflavin responsive multiple acyl-CoA dehydrogenase defect. Pediatr Res 16:861-868

19. Gregersen N, Mortensen PB. Kolvraa S 1983 On the biologic origin of C6C10-dicarboxylic and C6-C 10- $\omega-1$ Hydroxy monocarboxylic acids in human and rat with acyl-CoA dehydrogenation deficiencies: in vitro studies on the $\omega$-and $\omega$-1-oxidation of medium-chain (C6-C12) fatty acids in human and rat liver. Pediatr Res 17:828-834

20. Tanaka K 1979 Jamaican Vomiting Sickness In: Vinken PJ, Bruyn GW (eds) Intoxication of the Nervous System, Part II, vol 37. In: Handbook of Clinical Neurology. North Bay Publishing Company, New York, pp 529-535

21. Colle E, Mamer OA, Montgomery JA. Miller JD 1983 Episodic hypoglycemia with $\omega$-hydroxy fatty acids excretion. Pediatr Res 17:171-176 